\title{
Narrativa
}

narrativa

Nuova serie

39 | 2017

Nuove frontiere del Sud

\section{En Attendant Méditerranée}

\section{Franco Cassano}

\section{(2) OpenEdition}

\section{Journals}

\section{Edizione digitale}

URL: https://journals.openedition.org/narrativa/629

DOI: $10.4000 /$ narrativa.629

ISSN: 2804-1224

\section{Editore}

Presses universitaires de Paris Nanterre

\section{Edizione cartacea}

Data di pubblicazione: 1 décembre 2017

Paginazione: 13-27

ISBN: 978-2-84016-289-6

ISSN: 1166-3243

\section{Notizia bibliografica digitale}

Franco Cassano, «En Attendant Méditerranée», Narrativa [Online], 39 | 2017, online dal 01 décembre 2021, consultato il 14 janvier 2022. URL: http://journals.openedition.org/narrativa/629 ; DOI: https:// doi.org/10.4000/narrativa.629

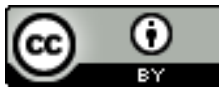

Narrativa est mise à disposition selon les termes de la Licence Creative Commons Attribution 4.0 International. 


\section{En Attendant Méditerranée}

\section{GLI ANNI DELLA SPERANZA}

Se è vero che una costruzione teorica non è mai riducibile al contesto storico nel quale è stata formulata, è anche vero che essa diventa difficilmente comprensibile se quel contesto viene rimosso. È impossibile, infatti, cogliere l'attenzione riscossa negli anni Novanta da quella produzione saggistica che individuava nel Mediterraneo il fulcro di una nuova prospettiva (geo-politica, geo-economica e geo-culturale) senza ricostruire, ovviamente in modo sommario, il clima di quel decennio, le paure e soprattutto le speranze che hanno fatto seguito alla caduta dell'URSS e del sistema socialista. La brusca chiusura del "secolo breve" imponeva infatti una riflessione impegnativa sui futuri possibili e furono in molti quelli che provarono a immaginare gli scenari nuovi che quel salto stava aprendo.

Il più celebre di questi tentativi è quello messo a punto nel 1992 da Francis Fukuyama1, che lo portò a proclamare la "fine della storia", ovvero la fine di un lungo percorso in cui l'umanità si era trovata divisa tra ideologie e modelli di vita contrapposti. Una volta finita la guerra fredda, che aveva assegnato con un verdetto inequivocabile lo scettro del vincitore al capitalismo liberal-democratico nella sua versione nord-americana, non aveva più senso inseguire un modello di vita alternativo. Il compito principale diventava quello di apprendere il più possibile dal paradigma vincente, adeguando le forme politiche-istituzionali ed economiche dei diversi paesi ai parametri del capitalismo liberale. Si apriva un mondo nel quale il primato dell'economia avrebbe permesso di neu-

1. Fukuyama, Francis, La fine della storia e l'ultimo nomo, Milano, Rizzoli, 1992. Ma sul percorso successivo di Fukuyama si veda l'acuta ricostruzione di Anderson, Perry, American Foreign Policy and Its Thinkers, London - New York, Verso, 2017. 
tralizzare i conflitti radicali, avendo trovato nella crescita continua il pharmakon decisivo, lo strumento per il loro superamento. Per la verità nello stesso campo liberale questa lettura lineare e irenistica di Fukuyama aveva trovato un vigoroso contraltare polemico in un altro studioso americano, Samuel Huntington, che, lungi dal vedere il futuro come un rassicurante mondo unipolare, sottolineava il rischio che la fine dell'esperienza socialista, segnando anche la fine del lungo primato della cultura dell'Occidente, potesse aprire la strada al pericolo di un conflitto tra le diverse civiltà2.

In quegli anni si sono affermate però anche altre letture delle possibilità aperte dal nuovo quadro planetario emerso dalla caduta del socialismo sovietico. La più vicina a quella di Fukuyama è stata sicuramente la prospettiva della "terza via", che ha trovato in Europa e soprattutto in Tony Blair il suo maggiore esponente. Il cuore teorico-politico di quella prospettiva, supportata dal lavoro di Anthony Giddens e Ulrich Beck ${ }^{3}$, era la proposta di dar vita a un nuovo riformismo, capace di condurre la sinistra oltre lo statalismo, che era stato il tratto comune all'esperienza socialdemocratica e a quella comunista ed era legato alla "guerra di posizione" e alla competizione politica tra i due blocchi ${ }^{4}$. Il nuovo mondo cosmopolita e la competizione globale imponevano di spingere i paesi più "avanzati" verso un nuovo dinamismo economico e sociale, verso una stagione di politiche "attive", capace di andare oltre l'orizzonte dei "trenta gloriosi", una trasformazione dei sistemi di protezione sociale, il passaggio dalla difesa del "vecchio" stato sociale universalistico alla costruzione di un welfare "positivo", fondato sulla "mobilitazione responsabile" dei cittadini ${ }^{5}$.

Le nuove prospettive non erano però limitate alle varianti di destra e di sinistra del campo liberale, ma nascevano anche all'esterno di quell'orizzonte, pur muovendo da premesse teoriche non sempre convergenti. Ciò che le accomunava era la convinzione che il nuovo scenario fosse più complesso e plurale di quello

2. Cfr. Huntington, Samuel P., Lo scontro delle civiltà e il nuovo ordine mondiale, Milano, Garzanti, 1997.

3. Beck, Ulrich, Giddens, Anthony, Lash, Scott, Reflexive Modernization. Politics, Tradition and Aesthetics in the Modern Social Order, Stanford, Stanford University Press, 1994; GidDens, Anthony, La Terza Via. Manifesto per la rifondazione della socialdemocrazia, Prefazione di R. Prodi, Milano, Il Saggiatore, 1999.

4. Nella ricostruzione di questo passaggio è particolarmente prezioso il lavoro di Giovanni Arrighi (Arrighi, Giovanni, Silver, Beverly J., Caos e governo del mondo. Come cambiano le egemonie e gli equilibri planetari, Milano, Bruno Mondadori, 2003; ARrighi, Giovanni, Il lungo XX secolo, Denaro, potere e le origini del nostro tempo, Milano, Il Saggiatore, 2014).

5. Cfr. Giddens, Anthony, Oltre la destra e la sinistra, Bologna, Il Mulino, 1997. 
rappresentato dal trionfalismo di Fukuyama, che indicava ai popoli come unica meta l'imitazione-inseguimento del modello americano. Secondo tali prospettive, che venivano da un retroterra teorico messo a fuoco negli anni Ottanta (gli anni del post, in cui un relativismo morbido sembrava presagire l'indebolirsi delle vecchie opposizioni ideologiche), il crollo sovietico non era né un trionfo da festeggiare né un lutto da celebrare, ma semplicemente l'ingresso in un'epoca post-moderna e post-occidentale, contrassegnata dalla pluralità delle differenze culturali, che avrebbe messo fine al monologo coloniale degli ultimi secoli.

In questo quadro il Mediterraneo, lungi dall'essere il luogo dell'arretratezza da ricondurre all'ordine economico dominante o da abbandonare al degrado, diventava il centro di una visione del mondo alternativa, capace di battere tutti i fondamentalismi, compreso quello della crescita ininterrotta e dell'individualismo competitivo che costituiva il nucleo costitutivo dell'identità dei vincitori. L'idea era di avviare la costruzione di una koinè culturale/politica, una convivenza paritaria tra le culture che provasse ad associare i popoli affacciati su quel mare. Questo incontro avrebbe permesso altresì di contrastare la deriva dell'Unione Europea, che, pur essendo nata dal superamento di secolari contrapposizioni, stava però, a causa dei nuovi equilibri prodotti dell'unificazione tedesca, sempre più rinchiudendosi sul suo cuore continentale, incapace di vedere nei paesi mediterranei qualcosa di diverso da una periferia perennemente imperfetta e recalcitrante rispetto ad un centro virtuoso e produttivo. La convinzione comune a molti studiosi era invece quella di poter fare del Mediterraneo un luogo post-coloniale, non l'archetipo delle contrapposizioni identitarie e delle ostilità, un vallum valicabile solo con le conquiste militari, ma il luogo della "misura", dove era possibile decostruire le tentazioni di ogni fondamentalismo, riprendendo il filo delle mille contaminazioni e dei mille ibridi nati sulle sue sponde.

Tra le prospettive più radicali emerse in quegli anni vale la pena di ricordare anche quella della "decrescita felice" proposta da Serge Latouche ${ }^{6}$, che, leggendo con una certa disinvoltura la cultura occidentale come univocamente dominata da una mega-macchina produttivistica, proponeva di attaccare l'cidolo" della crescita, reincorporando l'economia, sottrattasi a ogni vincolo

6. Latouche, Serge, La megamacchina. Ragione tecnoscientifica, ragione economica e mito del progresso, Torino, Bollati Boringhieri, 1995. 
esterno e divenuta auto-normativa, nel reticolo dei rapporti sociali, sulla scia di un'intuizione già presente nell'opera di Karl Polanyi'.

Su un versante per certi versi opposto, nel campo delle prospettive radicali va ricordata anche quella formulata da Antonio Negri e Michael Hardt in Impero ${ }^{8}$, che aveva invece l'ambizione di proporre una nuova versione dell'antica fiducia marxiana nelle virtù rivoluzionarie del capitalismo. Se è vero che quest'ultimo, ormai governato dalla finanza transnazionale, stava producendo su scala planetaria lo sradicamento degli uomini, è anche vero che tale processo non era, come lamentavano in tanti, solo un turbine distruttivo. In realtà la de-territorializzazione selvaggia avviata da un capitale imperiale e senza patria assolveva un compito progressivo, perché cancellando le anguste divisioni religiose e nazionali, apriva la strada all'avvento del nuovo proletariato del XXI secolo, la moltitudine, un soggetto, in verità, molto più indefinibile e ambiguo (macchine desideranti o comunità francescana?) del suo antenato marxiano.

Quel decennio fu dunque un passaggio più ricco di possibilità dell'immagine che oggi ne conserviamo, un periodo non dominato da una sola narrazione, in cui il futuro apparve a molti, pur se diversi e talvolta in conflitto tra loro, più aperto e promettente di quanto non ci appare oggi. Se ad Alain Minc era addirittura parso possibile parlare improvvidamente di "globalizzazione felice", va ricordato che anche le posizioni più radicali sembravano riconoscere al capitalismo la conquista di una capacità espansiva continua. Anche chi criticava 1" occidentalizzazione del mondo"10 riconosceva di trovarsi di fronte a un meccanismo, che, dominato dalla tecnica, si era messo alle spalle insieme alle crisi tutti gli ostacoli, a un "uni-versalismo" convinto di poter modellare sulla propria misura il multi-verso del mondo, comprese le vecchie divisioni nazionali o religiose.

Ma come sempre la storia si sarebbe mostrata più ruvida, complessa e irrispettosa delle filosofie che cercavano di inquadrarla.

7. Polanyi, Karl, La grande trasformazione, Torino, Einaudi, 1974. La prospettiva della decrescita, per quanto arricchita nel tempo da una serie di aggettivi rassicuranti (felice, serena, conviviale, sostenibile, ecc.), non ci sembra abbia la forza di reggere il confronto con la realtà di un mondo interconnesso e inquieto come quello in cui viviamo.

8. Hardt, Michael, Negri, Antonio, Impero. Il nuovo ordine della globalizzazione, Milano, Rizzoli, 2000.

9. Minc, Alain, La Mondialisation heureuse, Paris, Plon, 1997.

10. Latouche, Serge, L’occidentalizzazione del mondo, Torino, Bollati Boringhieri, 1992. 


\section{IL NUOVO SECOLO}

Il primo manifestarsi clamoroso di tale discontinuità, il momento dell'esplosione di una pressione accumulatasi nel tempo ha una data precisa: l'11 settembre. Lo spietato e spettacolare attentato costituisce una svolta traumatica perché rappresenta il riaffacciarsi della faccia tragica e inconciliabile della storia, una faccia che si riteneva fosse ormai alle spalle, quella del conflitto radicale tra le differenze. L'attacco alle Twin Towers non costituisce infatti solo una formidabile smentita di ogni irenismo, ma segna l'emergere di un protagonista internazionale inaspettato, il fondamentalismo religioso, la riaffermazione militarizzata su scala globale di uno scontro identitario, in contrasto con l'opinione diffusa che riteneva che, grazie alla forza secolarizzata del capitalismo globale, le pretese "essenzialistiche" delle identità stessero progressivamente indebolendosi. Il trauma rappresentato dall'attentato segna l'inizio di un crescente allontanamento delle rive del Mediterraneo, l'avvio di una spirale che, esaltando i meccanismi di difesa immunitari di ogni cultura, tende a rinchiuderla nella versione più povera e feroce di se stessa, respingendo ai margini le versioni più aperte $\mathrm{e}$ tolleranti. Il gioco è cambiato: ora esso è passato, con intensità e tempi diversi, in mano ai "duri" di ogni schieramento, quelli per i quali la pace è raggiungibile solo sulla base dell'azzeramento dell'altro. Le differenze, lungi dall'esaurirsi nel grande patchwork postmoderno, sono tornate ad essere anche la giustificazione delle guerre.

Dal canto suo l'Unione Europea, lungi dal contribuire a quel processo di avvicinamento, ha manifestato tutte le sue difficoltà. Le politiche di austerity anche quando non sono riducibili, come vuole una facile letteratura, a una pura manovra dei "poteri forti", sono state il cavallo di Troia attraverso il quale l'egemonia tedesca sulla nuova costruzione si è insediata fino a sacrificare ad essa ogni innovazione. E se è giusto riconoscere alla Germania la capacità di aver dato per tempo risposte rigorose e previdenti alla nuova fase della concorrenza internazionale, è impossibile non notare come i suoi successi economici abbiano finito per spostare l'asse di gravità dell'Unione, proponendo implicitamente a quest'ultima il proprio modello, l'Ordoliberalismus ${ }^{11}$, come unico e indiscutibile.

Tale ossessione delle politiche di equilibrio di bilancio ha però accentuato le differenze tra i vari paesi anziché ridurle. Si è venuta così affermando un'idea

11. Sul ruolo dell'Ordoliberalismus nella crisi dell'Unione Europea, si vedano le osservazioni di BECK, Ulrich, in Europa tedesca. La nuova geografia del potere, Roma-Bari, Laterza, 2013. 
dell'Europa che sublima la gerarchia di potenza tra gli stati in una contrapposizione morale tra le virtù luterane e il lassismo dei paesi del sud. Una volta trasformata una differenza in una gerarchia, la voce di chi è più debole non è credibile. È l'eclisse del Mediterraneo dalle politiche europee. Se negli anni Novanta esso aveva recitato un ruolo minore, ma pur sempre visibile (si pensi al partenariato avviato tra il 1995 e il 1996 a Barcellona) adesso sembra essersi avviato un completo rovesciamento della sua immagine: mare monstrum, un abisso impassibile che ingoia $\mathrm{i}$ migranti che cercano di attraversarlo, fonte di caos se non via del terrore.

La stagione delle cosiddette "primavere arabe", che all'inizio di questo decennio sembrava aver offerto all'Unione Europea una grande occasione, l'ha vista oscillare tra il disinteresse tedesco e i rigurgiti neo-coloniali anglo-francesi, che hanno portato all'intervento "umanitario" in Libia, manifestando la medesima cecità dell'intervento in Irak, e dando l'ennesimo contributo al rilancio del fondamentalismo islamico. Tale atteggiamento, insieme reticente e intrusivo, nei riguardi delle primavere arabe ha manifestato tutte le debolezze della visione europea del Mediterraneo, divisa tra il disinteresse cinico, l'interventismo coloniale, e, nel migliore dei casi, una solidarietà sincera, ma spesso incapace di cogliere il quadro complesso che caratterizzava quei moti e che in essi riusciva a vedere solo l'immagine riflessa di se stessa ("aiutiamoli a diventare come noi!’). In questo modo si è affermata una vera e propria spirale regressiva: sulla sponda sud i protagonisti, peraltro in conflitto tra loro, con l'unica e preziosa eccezione della Tunisia, sembrano essere diventati i capi militari e i fondamentalisti, su quella nord l'esodo dei migranti miscelandosi con le paure innescate dalla crisi ha prodotto quell'ostilità verso chi fugge da altri mondi che vediamo riaffacciarsi in diversi paesi europei. Ancora una volta la coppia paura/divisione sembra mettere in scacco la coppia opposta, quella speranza/solidarietà.

Quel mare tra le terre, che nel corso degli anni Novanta sembrava aver conquistato uno spazio egemonico crescente, di fronte al terrorismo integralista e all'"esportazione neo-con della democrazia", ha visto infittirsi il numero dei suoi nemici. E adesso a chi vuole tornare a ridurre la distanza tra le rive tocca prendere una rincorsa più lunga. La battaglia, tuttora decisiva per l'Europa mediterranea, non è persa, ma si sono spostati i rapporti di forza.

\section{LA MONDIALIZZAZIONE INFELICE}

Per orientarci rispetto alle grandi trasformazioni in corso non basta tuttavia ricordare lo scarto tra le aspettative di quegli anni e ciò che è seguito, chiudersi nella delusione. Occorre imparare dagli avvenimenti, allargare lo sguardo, 
provare a ricostruire, anche se in modo rapido e sommario, i principali mutamenti che sono intervenuti fino a sfociare nel nostro presente. Formuleremo alcuni punti, ovviamente senza pretese di esaustività, per sottolineare i passaggi più rilevanti.

a) In primo luogo è impossibile non riconoscere che l'espressione coniata nel 1997 da Alain Minc, "mondializzazione felice”, sembra oggi appartenere a un'epoca remota. E questo non solo a causa dell'11 settembre e della feroce spirale ritorsiva che abbiamo descritto. A partire dal 2006, con il crollo dei titoli subprime a Wall Street, una crisi economica parte dagli Stati Uniti finendo per coinvolgere tutti i paesi avanzati e in particolare quelli europei. Dalle borse alle banche il circuito finanziario mostra le sue debolezze e il suo lato oscuro, il cinismo sistematico e l'avventurismo che lo animano. Il nuovo capitalismo globale e il progetto neo-liberale adesso non s'imbattono solo nella determinazione di un nemico "esterno", ma scoprono all'improvviso che ne esiste uno interno, che la spregiudicatezza dei padroni del mercato è accompagnata da vicino dall'ombra del crollo.

La crisi è grave perché colpisce i mercati, ma anche e soprattutto perché apre una profonda crisi di legittimità, incrinando la credibilità stessa dell'idea di un'espansione continua, che riteneva di aver trovato la formula magica per dare una chance a tutti. I crack finanziari non comportano solo il dilagare dell'incertezza e l'inasprimento delle disuguaglianze, ma minano la fiducia che sia possibile allargare all'infinito questo gioco, espandendo irreversibilmente la formazione di un ceto medio organico ai meccanismi del neoliberismo. È l'egemonia di un modello che inizia a mostrare le sue crepe. La mobilità sociale e il nuovo individualismo che avevano scaldato i cuori del riformismo, il nucleo egemonico della sua narrazione e della rivoluzione passiva che la accompagnava, appaiono illusori o comunque limitati. Gli ascensori sociali non funzionano più; sempre più spesso rimangono fermi al piano quando non scendono a quelli inferiori. La crescita delle disuguaglianze non è più addolcita dalla speranza di poterne scalare almeno in parte l'edificio. S'incrina in profondità l'espansione continua di quel ceto medio che aveva sostenuto il sogno del neoliberismo e della mondializzazione felice. Non è un caso che al libro di Minc abbia replicato in tempi recenti, probabilmente con uno schematismo opposto e simmetrico, Thomas Guénolé con il suo La mondializzazione infelice ${ }^{12}$.

12. GuÉnOLÉ, Thomas, La Mondialisation malheureuse. Inégalité - Pillage - Oligarchie, Paris, First, 2016. 
b) Il secondo punto su cui porre l'accento è la diaspora dei sud. Questi ultimi, o forse dovremmo dire quei paesi che a lungo erano stati chiamati "arretrati", lungi dal convergere nella costruzione di una forma di vita alternativa al monoteismo della crescita, hanno percorso, nei quasi trent'anni passati dalla caduta dell'impero sovietico, strade molto diverse. Una parte di essi ha subito l'urto più duro dei processi di globalizzazione ed è crollata a seguito del saccheggio delle risorse, delle crisi ambientali e delle guerre civili. Le colonne infinite dei migranti rappresentano l'esodo da questi "paesi falliti" risucchiati dal disastro economico, istituzionale e politico. E se è sicuramente troppo semplice rinviare tutte le cause di quel disastro alla spietatezza delle élites finanziarie prosciogliendo comodamente altri responsabili, è fuori discussione che per quei paesi il salto nell'economia globale sia stato sinonimo di tracollo e divisioni. Se nel vecchio mondo bipolare essi potevano ancora contare sulla complicità e gli aiuti interessati dei due poli rivali, oggi sono abbandonati a se stessi, anche se si delinea l'attivismo di nuovi global players come la Cina.

Accanto ai paesi che franano ci sono però anche quelli di successo, quelli entrati come protagonisti nel cerchio dello sviluppo capitalistico, con l'obiettivo del raggiungimento dei livelli di benessere che fino a poco tempo fa costituivano una prerogativa irraggiungibile dei paesi occidentali. I modelli di sviluppo che si sono venuti affermando comportano sicuramente costi sociali e ambientali altissimi, ma tali costi non sembrano (o almeno non sembrano ancora) mettere in discussione la rincorsa. Non sappiamo se il XXI secolo sarà, come molti dicono, il secolo cinese o asiatico, ma sicuramente l'Oriente sta diventando un protagonista della storia futura. La calamita dello sviluppo, del benessere e dei consumi sembra aver vinto in più parti del globo la battaglia; i paesi più ricchi sono la meta geografica dei migranti dei paesi in rovina, ma, almeno sul piano dei consumi e del reddito, anche la meta simbolica dei paesi-continente, di grandi masse che mirano a raggiungere standard di vita più alti. La ferocia del terrorismo deriva anche dalla rivolta contro questa dinamica, insinuandosi nei suoi vuoti e nelle sue ingiustizie.

c) C'è però un'importante novità che discende da questo lato della globalizzazione: i paesi emergenti sono quelli che, facendo dei loro bassi salari un vantaggio competitivo rispetto a quelli più ricchi, hanno avviato una concorrenza nuova e serrata nei riguardi delle economie e delle conquiste dei lavoratori di quei paesi. Se i paesi più avanzati non saranno capaci di rinnovarsi e rispondere a questa sfida sono destinati a scendere nelle gerarchie mondiali e tutti gli equilibri sociali da loro faticosamente costruiti sono destinati a deteriorarsi. È probabilmente l'effetto più dirompente di questo passaggio di secolo: l'attacco al 
welfare state e ai diritti conquistati in Occidente da parte del capitale finanziario non avviene, come dice una narrazione semplificata, attraverso una strategia frontale. Il metro rimane sempre quello della convenienza e dei profitti, ma passa attraverso la costruzione di una sorta di alleanza con le classi dirigenti e con i nuovi ceti medi di quei paesi che fino a pochi decenni fa rientravano nella categoria dei paesi arretrati. Centinaia di milioni di esseri umani si sono messi in movimento, ma il cuore di questa ascesa ruota intorno alla concorrenza e alla divisione tra i lavoratori dei diversi paesi, alcuni dei quali offrono ai capitali condizioni per gli investimenti molto più appetibili di quelle proposte dai paesi "ricchi". L'internazionale dei profitti ha sconfitto l'internazionale dei lavoratori, lanciando il suo attacco in modo indiretto, ma efficace, contro le conquiste prodottesi nei paesi più ricchi, rappresentandole come se esse fossero delle rendite da colpire.

Non è difficile riconoscere che quel fenomeno che nel pensiero meridiano veniva chiamato "integralismo della corsa" 13 , la concorrenza serrata e pervasiva, invece di ridursi si è allargato coinvolgendo nel proprio gioco paesi con tradizioni diverse da quella dell'Occidente, che, lungi dall'opporsi frontalmente allo sviluppo capitalistico, lo hanno adottato e rilanciato come strumento della propria affermazione e rivalsa contro il secolare predominio euro-americano. In poche parole la competizione non ha allentato la sua morsa, ma l'ha moltiplicata assumendo forme nuove e complesse, in cui economia e politica s'intrecciano anche grazie all'iniziativa dei paesi emergenti. Non è vero che la globalizzazione coincida in modo lineare con la morte della politica. I paesi emergenti hanno avuto l'abilità di usare la politica a proprio favore. In modi diversi essa ha continuato a giocare un ruolo importante, lontano sia dalle attese messianiche sia dalle ortodossie dottrinarie del neo-liberismo ${ }^{14}$. Lungi dal cercare vie alternative le diverse tradizioni culturali hanno mostrato (non tutte nella stessa misura e con gli stessi successi) una grande duttilità, adottando il capitalismo e differenziandone le forme ben al di là dello schema weberiano, che sembrava vincolarlo in modo ferreo alla tradizione protestante. A differenza di quanto riteneva Fukuyama ci sono oggi molti capitalismi e l'alterità rispetto all'Occidente ha preso strade diverse da quelle previste. Come ha detto Giovanni Arrighi ${ }^{15}$, Adam Smith ha preso casa a Pechino.

13. Cfr. Cassano, Franco, Il pensiero meridiano, Bari-Roma, Laterza, 1996, pp. 67-78.

14. Sulla critica della visione neoliberista dello sviluppo si veda per tutti CHANG, Ha-Joon, Kicking Away the Ladder. Development Strategy in Historical Perspective, London, Anthem Press, 2003.

15. Arrighi, Giovanni, Adam Smith a Pechino. Genealogie della globalizzazione, Milano, Feltrinelli, 2007. 
Le differenze culturali hanno trovato vie d'impiego imprevedibili e non sembrano essersi "indebolite" né mescolate in un bazar postmoderno. È vero, le identità possono intersecarsi pacificamente costruendo un'umanità plurale, ma solo in determinate condizioni e soprattutto in assenza di disuguaglianze e differenziali di potere acuti che accentuano il sospetto, l'isolamento e l'ostilità. Il punto debole della leggerezza post-moderna è la sottovalutazione degli effetti che nei rapporti tra le dinamiche inter-culturali hanno avuto e hanno le disuguaglianze e i differenziali di potere ${ }^{16}$, della loro capacità di alimentare e alimentarsi di un sentimento di rivalsa nei riguardi delle umiliazioni subite. Da un lato questo risentimento anima i giovani immigrati di seconda generazione, che di fronte ad un mondo che esalta il successo individuale, ma riserva a molti solo l'emarginazione, trovano nel radicalismo il viatico simbolico per rovesciare il gioco e rappresentarsi come giustizieri per mandato divino. Dall'altro lato la fine del primato assoluto dell'Occidente libera sogni e desideri a lungo repressi. La frase di Georges Simenon, "un piccolo paese è un paese che è stato grande e se ne ricorda" 17 , non vale solo per i paesi che grandi sono stati, ma anche per quelli che immaginano di poterlo diventare. Insomma più che il delinearsi di un'alternativa corale e solidale agli imperativi del capitalismo hanno prevalso la logica dell'inseguimento e della competizione e la speranza di ribaltare antiche egemonie.

d) Tutti questi cambiamenti, che rendono il quadro complesso e imprevedibile, non devono però far perdere di vista un effetto politico comune di grande rilievo dei processi di globalizzazione. Intendiamo riferirci a quella contraddizione che alle linee di divisione classiche della politica, in primis quella tra destra e sinistra, aggiunge un nuovo cleavage, quello tra le classi e gli strati sociali favorevoli all'apertura dei mercati e alla concorrenza internazionale (élites finanziarie, imprese competitive sul mercato internazionale, professionisti, ricercatori, giovani qualificati inseritisi con successo nelle aree centrali), e quelli che invece, vedendo nella concorrenza globale soprattutto una minaccia, spingono per l'adozione di politiche di protezione (settori popolari colpiti dalla disoccupazione e dalla crisi, periferie sociali e territoriali, giovani disoccupati, ecc.). Fino a quando lo stato nazionale aveva mantenuto la sua centralità questa contraddizione è stata smorzata e ricomposta in secondo piano rispetto ai due schieramenti classici. Oggi la pressione della concorrenza globale, anche sotto la spinta

16. Sul peso esercitato da tali differenziali sulle relazioni tra le culture insiste CASSano, Franco, in "Necessità del Mediterraneo", in Cassano, Franco, Zolo, Danilo (a cura di), L'alternativa mediterranea, Milano, Feltrinelli, 2007, pp. 78-110.

17. Simenon, Georges, "Frontiere", in Limes, n. 2, 1994, pp. 289-296. 
dei paesi emergenti, e stanti i vincoli posti all'intervento dello Stato, rende quella contraddizione molto più forte, acutizzando le tensioni all'interno dei blocchi sociali che si riconoscevano nei tradizionali allineamenti politici. È anche da qui, da questo scollamento e incrinarsi della fiducia del ceto medio progressista formatosi nelle trente glorieuses ${ }^{18}$, che nasce quel fenomeno che chiamiamo con una sospetta pigrizia "populismo".

La destra si allontana dai settori più dinamici e "internazionalisti" della borghesia ma tale allontanamento viene compensato dalla simpatia crescente di vasti strati popolari, colpiti dalla disoccupazione e soprattutto dall'incertezza sul futuro, che vedono nella chiusura delle "vecchie" frontiere nazionali e nella difesa delle comunità tradizionali l'unica forma credibile di tutela. La sinistra è invece lacerata dalla tensione crescente tra i due imperativi che costituiscono la sua identità: da un lato la difesa degli interessi più deboli e dall'altro l'orizzonte internazionalista e cosmopolita. La difesa dall'insicurezza spinge a chiudersi, la fiducia nella propria capacità di misurarsi con le sfide spinge ad aprirsi. Oggi tale tensione sembra presentarsi nella forma di una crescente tensione tra due sinistre, e cioè, ci si perdoni lo schematismo, tra la sinistra dei diritti sociali (occupazione, difesa del welfare, comunitarismo) e la sinistra dei diritti civili (individualismo liberal, cosmopolitismo, mobilità) ${ }^{19}$.

Non è facile prevedere se questo processo riuscirà a produrre il superamento del vecchio cleavage destra/sinistra con quello del sovranismo/cosmopolitismo. Dato il carattere debole ed incerto della ripresa economica, più che un superamento e un nuovo equilibrio noi vediamo una crescente e pericolosa instabilità. Ma già oggi quella contraddizione ha prodotto una crisi su scala internazionale della sinistra, accentuando la divisione tra la sua ala moderata, costantemente tentata dal governo e dalla collaborazione con i dirimpettai moderati e "moderni" dell'altro schieramento, e quella radicale e intransigente, fatta più di passato che di futuro. Mentre l'internazionalismo sembra egemonizzato dallo spirito rapace del grande capitale, la difesa dei più deboli appare infettata dal nazionalismo e dalla xenofobia. Non sarà facile schiodarsi

18. Cfr. Fourastié, Jean, Les Trente glorieuses ou la Révolution invisible de 1946 à 1975, Paris, Fayard, 1979.

19. Le caratteristiche di questo processo di disarticolazione del blocco sociale della sinistra sono state descritte con lucidità da Nancy Fraser in "Il neoliberismo progressista contro il populismo reazionario: una scelta di Hobson", in GeISELBERGER, Heinrich (a cura di), La grande regressione. Quindici intellettuali da tutto il mondo spiegano la crisi del nostro tempo, Milano, Feltrinelli, 2017, pp. 61-71. 
da questa dinamica e provare a ribaltarla, ma è la prima cosa da fare, la direzione verso cui indirizzare e concentrare gli sforzi prima che sia troppo tardi.

\section{CHE FARE?}

Dalla rapida e sommaria carrellata appena formulata emergono novità che non potevano non avere un impatto forte sulla proposta del pensiero meridiano. In un tempo di crisi e di paura, di identità contrapposte che oscurano la percezione degli interessi comuni, la solidarietà e la speranza, che erano il presupposto epistemologico di quella proposta sono divenute risorse scarse. Esse continuano ad esistere e alimentano delle minoranze, ma mentre le minoranze degli anni Novanta potevano immaginare che il futuro le avrebbe premiate facendole diventare maggioranza, il tempo che viviamo si è dimostrato più avaro, premiando, come abbiamo visto, molto più i costruttori di muri che quelli di ponti.

Il significato ideale di quella proposta rimane inalterato, ma ha dovuto confrontarsi con le complicazioni prodotte da una storia impertinente e spesso indifferente alle nostre buone ragioni. Ma deprecare i tempi è una mossa ridicola ed inutile. È il momento invece dell'apprendimento, quello in cui la resistenza dei principi deve confrontarsi con un cammino irto di ostacoli vecchi e nuovi. Navigare è necessario, ma i venti che gonfiavano le nostre vele oggi sono molto meno favorevoli, spirano di traverso e spesso anche contro. Quelli che di fronte alle difficoltà rimangono a casa non ci interessano. Ma non sono pochi quelli per i quali apprendere significa cambiare destinazione, andare dove il vento ci porta, accettare che sia esso a decidere il nostro approdo. Per noi la meta rimane la stessa, anche se la rotta non si è rivelata né lineare né sicura. Occorre provare ad usare i venti al proprio fine, accettando il rischio di un percorso tortuoso che ci può far arrivare altrove o in nessun luogo. Ma questo è l'unico modo per andare avanti, sfidando sia l'indignazione di chi, se correggi la rotta per prendere il vento, ti accusa di opportunismo sia lo scetticismo di chi, sentendo dove pretendi di arrivare, ti compatisce sorridendo. La pigrizia intellettuale, l'abbiamo capito, è molto diffusa. Essa è duttile, feconda e immortale e talvolta si nasconde dietro il suo contrario. Ed è presente con una percentuale alta di esemplari, proprio come la stupidità ${ }^{20}$, in ogni aggregato umano.

20. Cfr. Cipolda, Carlo M., Allegro ma non troppo/Le leggi fondamentali della stupidità umana, Bologna, Il Mulino, 1988. 
Il viaggio da fare è lungo e non si può sapere quando verrà il tempo di vedere se la strada presa è stata quella giusta. Saranno altri, parlando dal loro presente, a giudicare. E poi saranno giudicati anche loro da quelli che verranno dopo. Ma c'è un apprendimento tra gli altri, il più doloroso e il più necessario, che non si può rimandare. Con un'espressione concisa possiamo dire che gli uomini si sono rivelati più deboli e limitati di quello che un'immagine generosa e forse giovanile portava ad aspettarsi da loro. A un disegno ambizioso che mirava alla costruzione di una fraternità più larga, sembrano preferire mete più ristrette, ma, almeno in apparenza, più concrete. Tale rappresentazione pessimistica dell'uomo è sicuramente unilaterale, ma può aiutare a contrastare il polo opposto e simmetrico, quell'ottimismo antropologico che ritiene che i limiti degli uomini siano l'effetto di un'imposizione esterna, di una congiuntura perfida di cui ci si può liberare una volta per tutte. Essi invece tornano continuamente a galla, sorprendendo e disorientando chi li riteneva affondati una volta per sempre. Si tratta quindi di riconoscere quei limiti, rispettando la complessità dell'animo umano, di provare a tenere insieme l'ottimismo della volontà e il pessimismo della ragione.

A questa espressione di Romain Rolland, resa famosa da Antonio Gramsci' ${ }^{21}$, se ne può affiancare, per corroborarla, un'altra, proveniente dalla tradizione liberale. Nella Prefazione ai suoi Saggi di scienza politica in Italia Norberto Bobbio dice: "Chi volge il pensiero scientifico a progetti di riforma della società" deve sapere "che la sua posizione è difficile ed è anche la più controversa: deve muoversi continuamente tra due insidie, tra la lezione dei cinici e il catechismo degli illuminati" ${ }^{22}$. E, se si vuole ritrovare lo stesso pensiero in altre tradizioni, si può ricordare quanto dice Reinhold Niebuhr:

I "figli della luce" devono armarsi della saggezza dei figli delle "tenebre", ma rimanere liberi dalla loro malvagità; essi devono conoscere il potere dell'egoismo nella società umana senza dargli giustificazione morale. Essi devono possedere questa saggezza al fine di ingannare, deviare, imbracare e limitare l'egoismo, individuale e collettivo, per il bene della comunità ${ }^{23}$.

21. Cfr. Gramsci, Antonio, Quaderni del carcere II, Torino, Einaudi, 1975, p. 1131.

22. BobBio, Norberto, Saggi sulla scienza politica in Italia, Roma-Bari, Laterza, 2005, p. XVI.

23. Niebuhr, Reinhold, Figli della luce e figli delle tenebre. Il riscatto della democrazia e critica della sua difesa tradizionale, Roma, Gangemi, 2002, p. 74. 
Oggi la strada giusta è quella che mira, con umiltà, a combattere la paura facendo spazio allintelligenza delle cose, anche se talvolta essa può dare l'impressione di essere tornati indietro rispetto ai momenti migliori. Due piccoli esempi, proprio partendo dal Mediterraneo: bisogna aiutare la Tunisia, l'unico paese che dopo la rivoluzione è riuscito a mantenere un assetto istituzionale democratico e rappresentativo. È un compito che non comporta clamori, ma lealtà e costanza. Emoziona meno delle piazze piene, ma è ugualmente prezioso perché assicura che quella spinta duri nel tempo. La manutenzione della democrazia contro i pericoli che nascono dalla paura è tutt'altro che un compito minore.

È necessario altresì aiutare la formazione di un governo stabile e unitario in Libia per riparare le rovine create e provare a costruire un'amicizia tra pari; un compito impervio e difficile, in un paese dove le divisioni tra le fazioni s'incrociano con le aspirazioni egemoniche delle grandi potenze, dove non è più permesso di sbagliare o di cadere nella tentazione di rinunziare o delegare ad altri. In realtà questi compiti piccoli spesso sono solo apparentemente tali. Invece della muscolarità superficiale degli "interventi umanitari", spesso tutt'altro che disinteressati, è necessaria un'intelligenza quotidiana, capace di aprire la strada a intese più larghe, che vada alle radici dei problemi, che scenda più a sud, affronti in modo deciso il problema dei "paesi falliti", pianti dove possibile il seme della fiducia, e inizi a ricomporre il puzzle di un Mediterraneo squassato dagli opposti fondamentalismi.

Ma per fare un passo in avanti in questa direzione è anche necessaria e urgente un'Unione Europea diversa da quella che abbiamo conosciuto, capace di una politica forte, meno soffocata dalla burocrazia e dalle ossessioni contabili. $\mathrm{Ci}$ sono molti modi, anche indiretti, per far sì che il Mediterraneo si trasformi da un'emergenza in una convenienza, e solo un'Europa diversa può assolvere questo compito. Ma perché questo accada è preliminare colpire duramente un luogo comune a cui abbiamo già accennato, molto diffuso nelle sue aree più forti e oggi divenuto quasi ufficiale. Oggi infatti va di moda la convinzione che l'affanno dell'Europa mediterranea sia da imputare esclusivamente ad essa, costituisca la conseguenza inevitabile della sua cultura. In questa lettura divenuta così popolare c'è una parte della verità, ma solo una parte di essa. Essa, infatti, non solo assolve le aree forti dai doveri di solidarietà (perché aiutare i sud se dissipano le risorse?), ma presenta rimozioni imbarazzanti. Viviamo nell'era nella quale gli stati nazionali hanno perso gran parte degli strumenti di sostegno allo sviluppo senza che l'Unione Europea abbia potuto o voluto riprodurli su scala più grande: il cosiddetto populismo èla conseguenza diretta di quest'assenza di un'Europa sociale. Un'assenza non casuale, perché nel vuoto della politica chi 
è forte diviene sempre più forte e chi è debole diviene sempre più debole. Chi vince attrae risorse, innescando una spirale positiva, una sequenza di successi; chi perde è risucchiato dalla spirale opposta, perde i migliori e più coraggiosi, che vanno altrove, e precipita verso la depressione e la passività. Il suo sistema immunitario s'indebolisce esponendosi a tutte le patologie della marginalità, in primis quella della malavita. E così il gioco è fatto: i sud, si dice in modo non del tutto disinteressato, hanno avuto ciò che si meritano. Decostruire questo pregiudizio dei forti è la premessa decisiva per cambiare l'Europa.

Se una politica sociale forte dell'Europa ci fosse stata, l'Unione oggi non sarebbe così malata e la fiducia dei suoi cittadini non sarebbe così logora. I politici di largo respiro non possono essere i ventriloqui delle aree forti, devono vedere più lontano dei think thank che li circondano. L'umiltà e la concretezza di cui abbiamo parlato non sono fini a se stesse, ma le articolazioni necessarie alla realizzazione di un disegno più grande. Il Mediterraneo ha bisogno dell'Europa, ma quest'ultima, se non vuole perdere se stessa e intende frenare le divisioni territoriali e nazionali, ha anch'essa bisogno del Mediterraneo.

Per provare a invertire la rotta sono necessarie qualità che sembrano tirare in direzioni opposte. Bisogna riuscire ad avere "un cuore intelligente" 24 , una fiducia nell'altro e nel futuro e una sapienza politica concreta, capace di invertire con pazienza tutte le spirali negative. Oggi è difficile tracciare una prospettiva forte e sicura, ma il futurismo dei globalisti e il passatismo dei nazionalisti non sono la soluzione, ma il problema. "Per ogni cosa c'è il suo momento": per tornare a sperare invece di scappare deprecandola davanti alla realtà, bisogna riconoscerla e trovare le vie e gli strumenti per governarla. Per strappare le differenze culturali alle loro patologie occorre inventare qualcosa che è necessario ma ancora non c'è. Perché la realtà apprenda dai sogni è necessario che i sogni abbiano appreso dalla realtà. E viceversa.

Franco Cassano Università di Bari

24. Rubiamo l'espressione al titolo del bel libro di Alain Finkielkraut (Un cuore intelligente, Milano, Adelphi, 2011) piegandola ovviamente al nostro discorso. 
